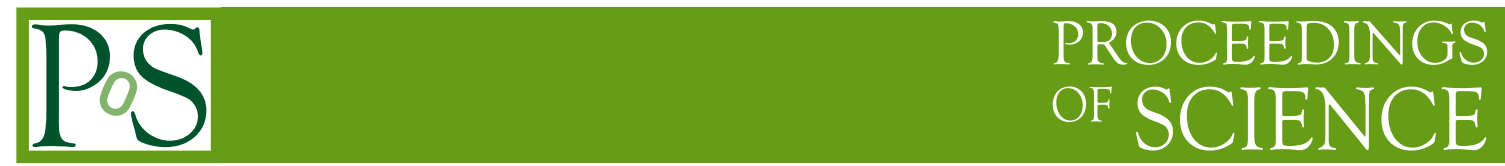

\title{
Dark sector searches in non-LHC experiments
}

\section{Jürgen Engelfried ${ }^{* \dagger}$}

Instituto de Física, Universidad Autónoma de San Luis Potosí, Mexico

E-mail: Jurgen.Engelfried@cern.ch

We will present recently published and soon to be expected results for dark matter searches in non-LHC experiments, namely BABAR, NA62, NA64, BELLE II, and PADME.

7th Annual Conference on Large Hadron Collider Physics - LHCP2019

20-25 May, 2019

Puebla, Mexico

* Speaker.

${ }^{\dagger}$ Thanks to the organizers for the invitation to give this presentation. 


\section{Introduction}

The need for an extension of the Standard Model (SM) - the dark sector - is well known and will not be discussed here. Introducing new particles and interactions depend on the type of fields used, and are usually separated into Scalars (also called "dark Higgs"), Axial particles called "Axions" and ALP's (Axion like particles), and new Vector Interactions, with a new vector boson called the "Dark Photon". Other possible candidates for dark sector particles are heavy neutral leptons (heavy neutrinos), and some other solutions.

In this presentation we will present recent already public results and expectations from nonLHC experiments which are already running or will start in the near future, namely from BABAR, NA62, NA64, Belle II, and PADME.

\section{Dark Photons}

A simple and natural extension of the SM is the introduction of a new interaction with a $U(1)$ symmetry [1,2], with the new boson, the "dark photon" $A^{\prime}$, interacting with the SM sector through a kinetic mixing

$$
\mathscr{L}_{\text {mix }}=-\frac{\varepsilon}{2} F_{\mu \nu}^{\mathrm{QED}} F_{\mathrm{dark}}^{\mu v}
$$

where $\varepsilon$ is the kinetic mixing strength. The smaller $\varepsilon$ the longer is the lifetime of the $A^{\prime}$.

If the $A^{\prime}$ is the lightest "Dark Sector" particle, it can only decay to SM particles, otherwise it can also decay in (lighter) dark sector particles.

For the experimental signatures and searches we have to distinguish two main cases: 1) The $A^{\prime}$ is the lightest "dark" particle and has a sufficiently short lifetime to decay into SM particles within the experimental setup, and 2) The $A^{\prime}$ is very long lived and/or decays into dark particles only and the experimental signature would be missing energy/momentum. For both cases there are recent experimental results which will be presented in the following.

2.1 BABAR: $e^{+} e^{-} \rightarrow \gamma A^{\prime}, A^{\prime} \rightarrow e^{+} e^{-}, \mu^{+} \mu^{-}$

The seminal study of BABAR in 2014 [3] searches for a short-lived $A^{\prime}$ produced together with a photon, decaying into a pair of electrons or muons. No signal is observed, and limits in the $\varepsilon-m_{A^{\prime}}$ plane are (see fig. 1 left): $\varepsilon \lesssim 10^{-3}$ for $0.02 \mathrm{GeV} / c^{2} \lesssim m_{A^{\prime}} \lesssim 10.2 \mathrm{GeV} / c^{2}$

2.2 NA64: $e^{-} Z \rightarrow e^{-} Z A^{\prime}, A^{\prime} \rightarrow e^{+} e^{-}$

A more recent search by NA64, a fixed target experiment at CERN with a $100 \mathrm{GeV} / c e^{-}$beam, probing lower $M_{A^{\prime}}$ masses and also smaller $\varepsilon$, looks for $A^{\prime} \rightarrow e^{+} e^{-}$[4], with special emphasis for $A^{\prime}=X$ with $m_{X}=16.7 \mathrm{MeV} / c^{2}$, the a new boson postulate to explain anomalies in ${ }^{8} \mathrm{Be}$ decays [5]. No signal is observed (see fig. 1 right).

2.3 BABAR: $e^{+} e^{-} \rightarrow \gamma A^{\prime}, A^{\prime} \rightarrow$ invisible

In this recent analysis [6] BABAR search for a long-lived $A^{\prime}$, with the experimental signature being a single photon and large missing momentum and energy. After observing no signal, the limits (see fig. 2 left) are $\varepsilon \lesssim 10^{-3}$ for a large mass range $m_{A^{\prime}}<8 \mathrm{GeV} / c^{2}$. 

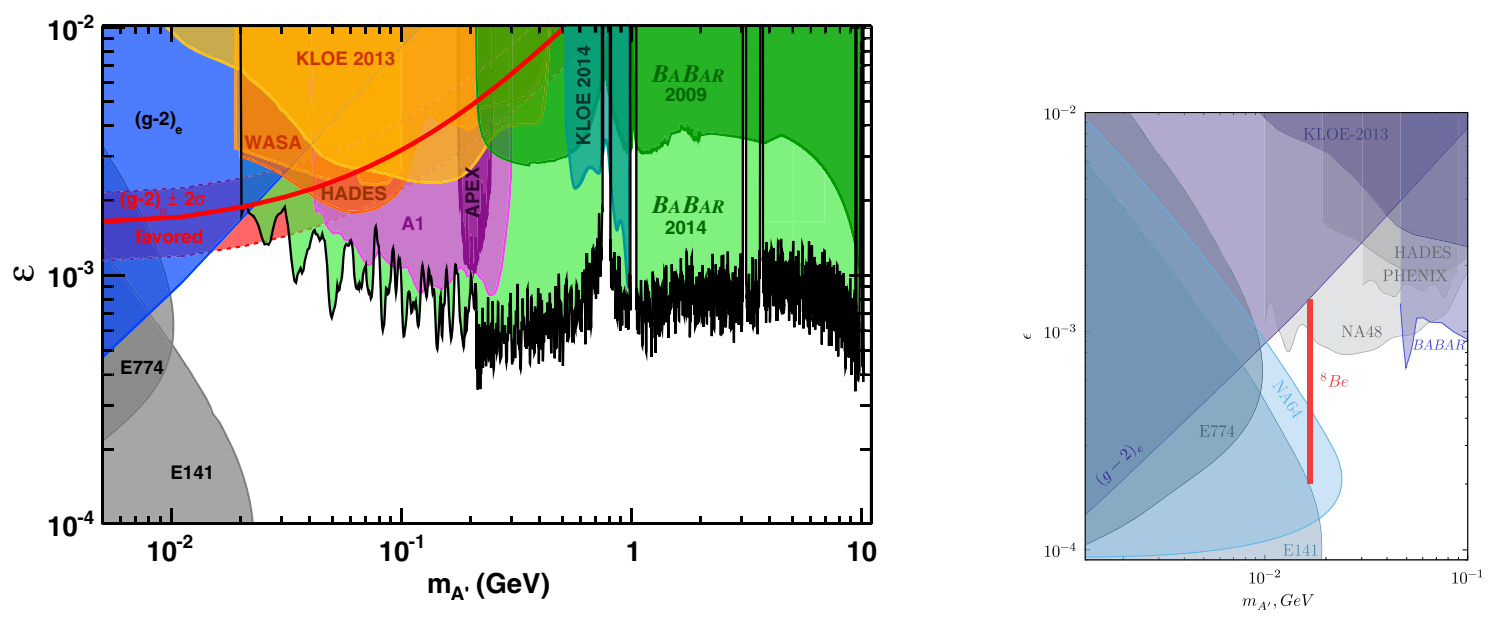

Figure 1: Limits on the kinetic mixing term $\varepsilon$ and the mass of the dark photon $m_{A^{\prime}}$ for a short-lived $A^{\prime}$. Left: BARAR [3]. Right: NA64 [4].
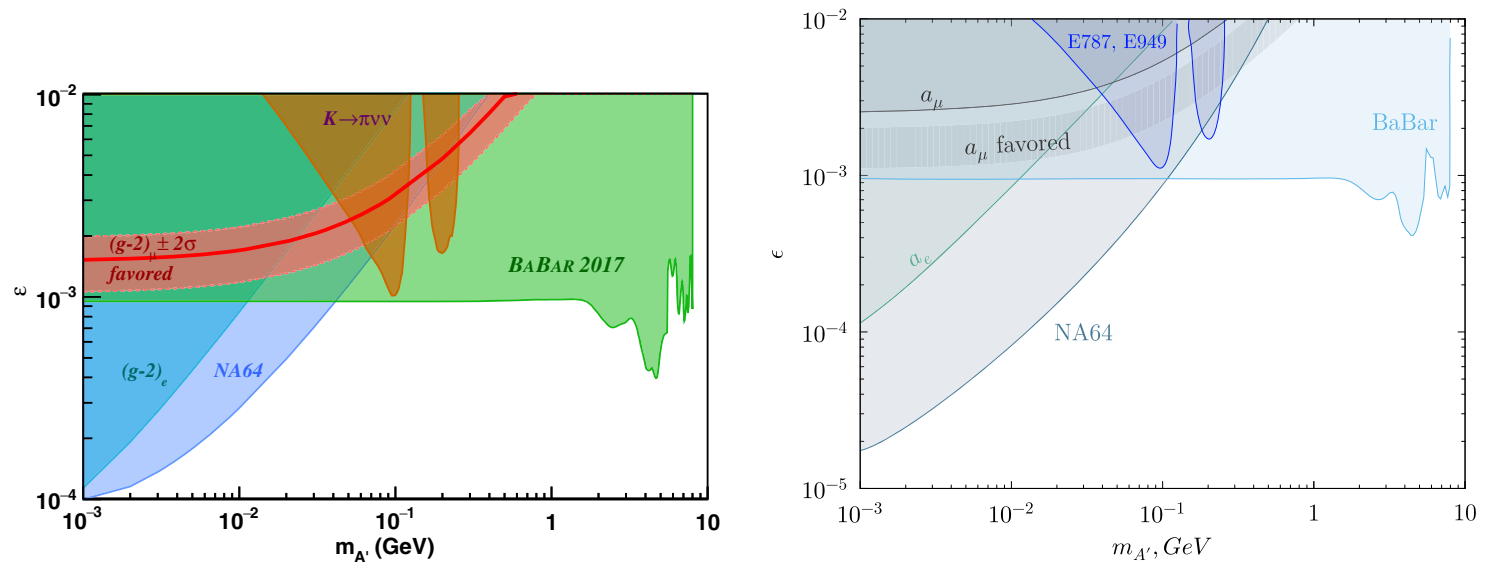

Figure 2: Limits on the kinetic mixing term $\varepsilon$ and the mass of the dark photon $m_{A^{\prime}}$ for a long-lived $A^{\prime}$. Left: BARAR [6]. Right: NA64 [7].

2.4 NA64: $e^{-} Z \rightarrow e^{-} Z A^{\prime}, A^{\prime} \rightarrow$ invisible

In a similar search, with the signature being a single electron and large missing energy, NA64 also searched [7] for a long-living $A^{\prime}$, setting limits (see fig. 2 right) for $10^{-5} \lesssim \varepsilon \lesssim 10^{-3}$ in a mass range $m_{A^{\prime}}<1 \mathrm{GeV} / c^{2}$.

2.5 NA62: $K^{+} \rightarrow \pi^{+} \pi^{0}, \pi^{0} \rightarrow \gamma A^{\prime}, A^{\prime} \rightarrow$ invisible

NA62, the rare kaon decay experiment at the CERN SPS, searched for a long-living $A^{\prime}$ via the reaction $\pi^{0} \rightarrow \gamma A^{\prime}, A^{\prime} \rightarrow$ invisible, with the $\pi^{0}$ 's coming from $K^{+} \rightarrow \pi^{+} \pi^{0}$ decays. The branching ratios are related via [8]

$$
\frac{\mathscr{B}\left(\pi^{0} \rightarrow \gamma A^{\prime}\right)}{\mathscr{B}\left(\pi^{0} \rightarrow \gamma \gamma\right)}=2 \varepsilon^{2}\left(1-\frac{m_{A^{\prime}}^{2}}{m_{\pi^{0}}^{2}}\right)^{3}
$$


NA62 [9] is designed to measure the branching ratio of $K^{+} \rightarrow \pi^{+} v \bar{v}$, in the SM predicted to be $8 \times 10^{-11}$. First results were already published [10] from the 2016 data taking period, and the status of the analysis of the 2017 data set are reported [11] at this conference.

The analysis profits heavily from the extremely high-efficiency photon veto system needed for the main measurement. The most abundant background is still due to $\pi^{0} \rightarrow \gamma \gamma$ where one $\gamma$ is not detected due to acceptance, inefficiency (very low), and photon conversions. The background is evaluated with data from a minimum bias trigger stream (independent of the signal stream). In the signal sample, a peak search in the $M_{\text {miss }}^{2}=\left(P_{K^{+}}-P_{\pi^{+}}-P_{\gamma}\right)^{2}$ distribution is performed.

For this analysis [12] only about $1 \%$ of the available data already on tape are used, leading to limits shown in fig. 3 .

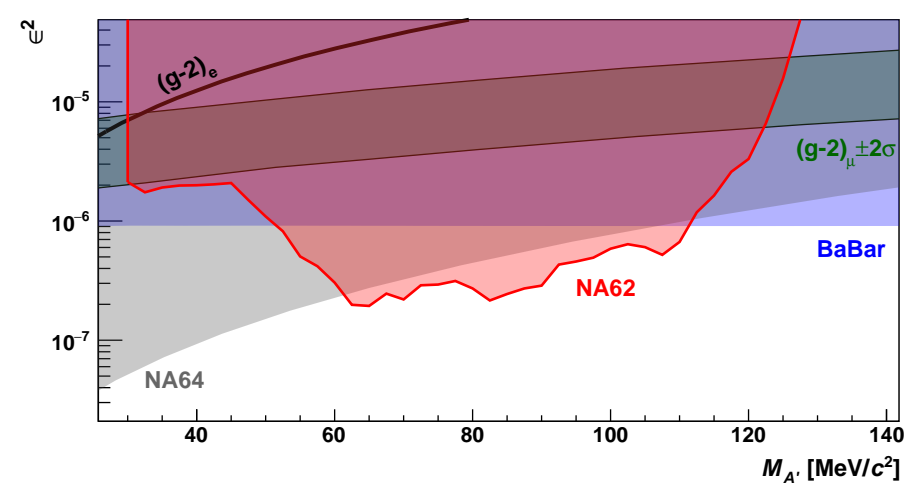

Figure 3: Limits on the kinetic mixing term $\varepsilon$ and the mass of the dark photon $m_{A^{\prime}}$ for a long-lived $A^{\prime}$ in $\pi^{0} \rightarrow \gamma A^{\prime}$ decays from NA62 [12]. Only about $1 \%$ of the available data on tape are used for this analysis.

\section{Heavy Neutral Leptons (neutrinos)}

NA62 searched for heavy neutral leptons $[13,14]$ in $K^{+} \rightarrow \mu^{+} v$ and $K^{+} \rightarrow e^{+} v$ decays. As shown in fig. 4 , a peak search is performed in the region of positive missing mass square, sufficiently far away from the SM peak at $m_{\text {miss }}^{2}=0$, which is used for normalization. No signal for a heavy neutral lepton is observed, and in fig. 5 the corresponding limits are shown.

\section{BABAR: Search for Stable Six-Quark State}

The completely symmetric six-quark state $u u d d s s$ could be deeply bound $[15,16]$. This is an extension of a prediction by Jaffe [17] in 1976, when he predicted the $H$ Dibaryon with a mass of $m_{H} \approx 2150 \mathrm{GeV} / c^{2}$. If $m_{H}>m_{P}+m_{e}+m_{\Lambda}=2055 \mathrm{MeV} / c^{2}$ the state should have a typical weak lifetime, while if $m_{S}<2055 \mathrm{MeV} / c^{2}$ the state $S$ should have a very long, cosmological lifetime; but if it is even deeper bound, $m_{S}<1878 \mathrm{MeV} / c^{2}, S$ cannot decay and will be stable, thus being an ideal candidate for a dark matter particle.

BABAR searched in $\Upsilon \rightarrow S \bar{\Lambda} \bar{\Lambda}$ [18], the signature being a missing mass recoiling against the $\bar{\Lambda} \bar{\Lambda}$ system. No signal was observed, and a limit of $\mathscr{B}\left(\Upsilon \rightarrow S \bar{\Lambda} \bar{\Lambda} \lesssim(1.2-1.4) \times 10^{-7}\right) @ 90 \%$ C.L. for the full mass range of $m_{S}<2.05 \mathrm{GeV} / c^{2}$ was set. 

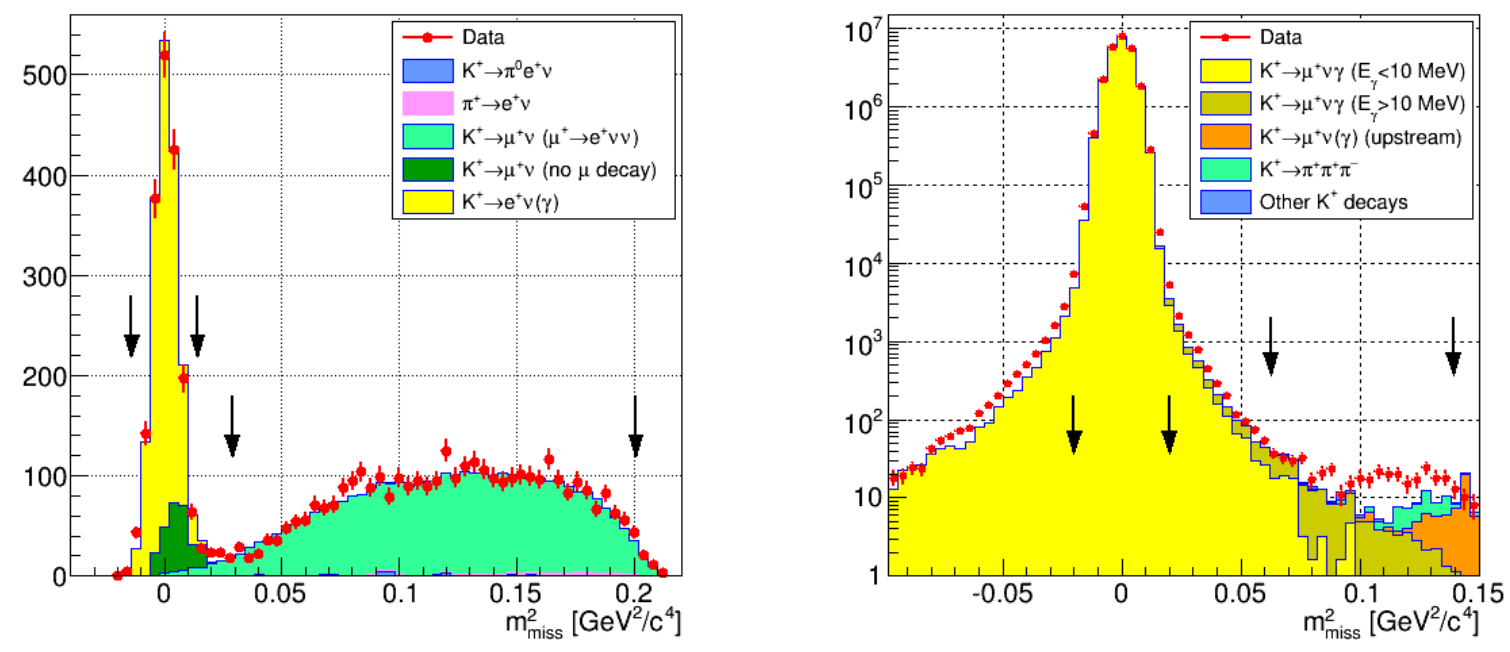

Figure 4: Distributions of $m_{\text {miss }}^{2}=\left(P_{K^{+}}-P_{l^{+}}\right)^{2}(l=e, \mu)$ for $K^{+} \rightarrow e^{+} v$ (left) and $K^{+} \rightarrow \mu^{+} v$ (right) decays. Arrows indicate the $m_{\text {miss }}^{2}$ regions used for normalization and where the peak search is performed. NA62 [14].

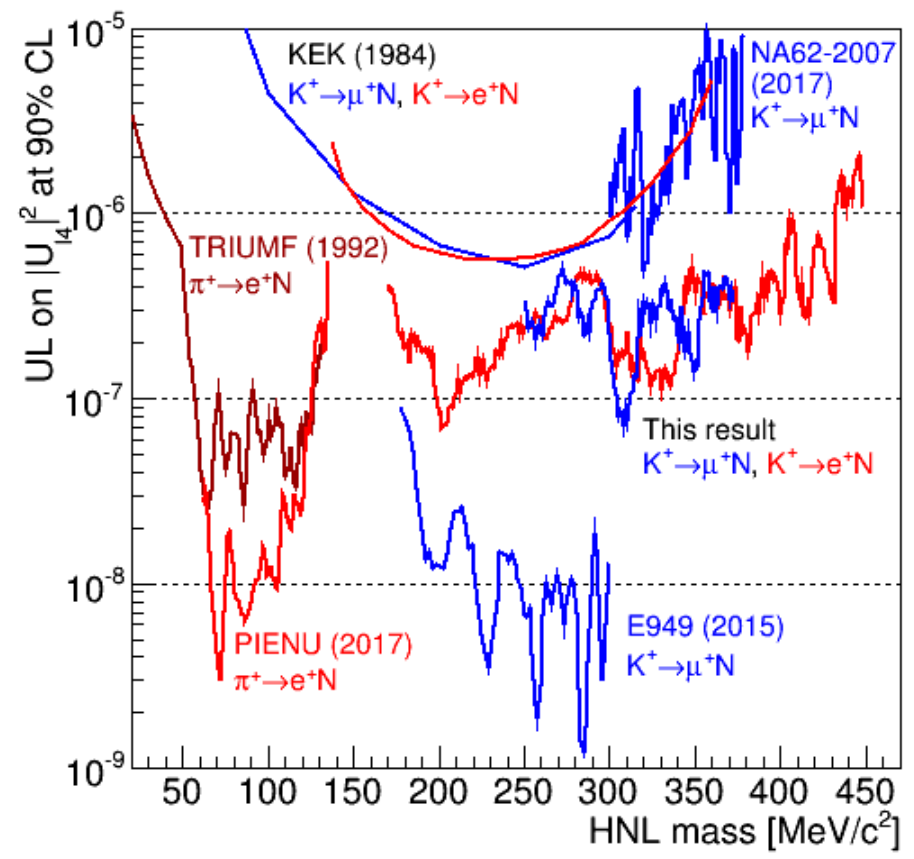

Figure 5: Limits on heavy neutral leptons from $K^{+} \rightarrow e^{+} v$ (red) and $K^{+} \rightarrow \mu^{+} v$ (blue) decays from NA62 [14] with the 2016 data set. 


\section{More results to come in the near future}

There are several experiments currently analyzing data, or just starting with data taking. We will not present projected exclusion plots here, we will better wait for real experimental results.

\subsection{PADME}

PADME [19] is a new fixed target experiment in Frascati. It took already some test data and is preparing for real data taking. They project [20] that limits for $A^{\prime} \rightarrow$ invisible will be improved for $m_{A^{\prime}} \lesssim 10 \mathrm{MeV} / c^{2}$, and $m_{A^{\prime}} \lesssim 100 \mathrm{MeV} / c^{2}$ after the experimental setup will be moved to Cornell and/or JLAB.

\subsection{BELLE II}

BELLE II [21] is just starting to take data, and they are well prepared for analysis with the experience from Belle and BABAR. For $A^{\prime} \rightarrow$ invisible they project [22] to improve the BABAR (see section 2.3) limit to $\varepsilon \gtrsim 5 \times 10^{-5}$ after acquiring $50 \mathrm{ab}^{-1}$, and also will search for ALP $\rightarrow \gamma \gamma$, expecting best limits in particular for the $1 \mathrm{GeV} / c^{2}<m_{\mathrm{ALP}}<10 \mathrm{GeV} / c^{2}$ mass range.

\subsection{NA64}

NA64 expects [23] up to $5 \times 10^{12}$ Electron on Target, and is also preparing to use a muon beam with up to $5 \times 10^{13}$ MOT, improving their existing limits for $A^{\prime} \rightarrow$ invisible.

\subsection{NA62}

NA62 takes data for dark searches in 2 modes: parasitically together with the main trigger for $K^{+} \rightarrow \pi^{+} v \bar{v}$, and during dedicated periods in Beam Dump Mode. During the 2016-2018 data taking period $\mathscr{O}\left(10^{16}\right)$ POT have been taken and are currently analyzed, and during 2021-2023 up to $\mathscr{O}\left(10^{18}\right)$ POT are expected. Analysis for $A^{\prime} \rightarrow e^{+} e^{-}, \mu^{+} \mu^{-}, \mathrm{HNL} \rightarrow$ visible, DarkScalar $\rightarrow$ visible, and ALP $\rightarrow \gamma \gamma$ for beam dump data are currently ongoing. Also analysis on the full statistics from 2016-8 for HNL searches in Kaon decays (section 2.5), and for $\pi^{0} \rightarrow \gamma A^{\prime}$ (section 3) are being performed.

\section{Acknowledgment}

I would like to thank Mauro Raggi (PADME), and Gerard Bonneaud and Fabio Anulli (BABAR) for providing material for this presentation.

\section{References}

[1] P. Fayet, Effects of the Spin 1 Partner of the Goldstino (Gravitino) on Neutral Current Phenomenology, Phys. Lett. 95B (1980) 285.

[2] B. Holdom, Two U(1)'s and Epsilon Charge Shifts, Phys. Lett. 166B (1986) 196.

[3] BABAR collaboration, Search for a Dark Photon in $e^{+} e^{-}$Collisions at BaBar, Phys. Rev. Lett. 113 (2014) 201801 [1406.2980]. 
[4] NA64 collaboration, Search for a Hypothetical 16.7 MeV Gauge Boson and Dark Photons in the NA64 Experiment at CERN, Phys. Rev. Lett. 120 (2018) 231802 [1803. 07748].

[5] A. J. Krasznahorkay et al., Observation of Anomalous Internal Pair Creation in Be8 : A Possible Indication of a Light, Neutral Boson, Phys. Rev. Lett. 116 (2016) 042501 [1504 . 01527].

[6] BABAR collaboration, Search for Invisible Decays of a Dark Photon Produced in $e^{+} e^{-}$Collisions at BaBar, Phys. Rev. Lett. 119 (2017) 131804 [1702.03327].

[7] NA64 collaboration, Search for vector mediator of Dark Matter production in invisible decay mode, Phys. Rev. D97 (2018) 072002 [1710.00971].

[8] B. Batell, M. Pospelov and A. Ritz, Exploring Portals to a Hidden Sector Through Fixed Targets, Phys. Rev. D80 (2009) 095024 [0906.5614].

[9] NA62 collaboration, The Beam and detector of the NA62 experiment at CERN, JINST 12 (2017) P05025 [1703.08501].

[10] NA62 collaboration, First search for $\mathrm{K}^{+} \rightarrow \pi^{+} v \bar{v}$ using the decay-in-flight technique, Phys. Lett. B791 (2019) 156 [1811.08508].

[11] N. Estrada-Tristán, Recent kaon decay results from NA62, in LHCP19, these proceeding, 2019.

[12] NA62 collaboration, Search for production of an invisible dark photon in $\pi^{0}$ decays, JHEP 05 (2019) $182[1903.08767]$.

[13] NA62 collaboration, Search for heavy neutrinos in $K^{+} \rightarrow \mu^{+} v_{\mu}$ decays, Phys. Lett. B772 (2017) 712 [1705.07510].

[14] NA62 collaboration, Search for heavy neutral lepton production in $K^{+}$decays, Phys. Lett. $\mathbf{B 7 7 8}$ (2018) 137 [1712.00297].

[15] G. R. Farrar, Stable Sexaquark, 1708.08951.

[16] G. R. Farrar, 6-quark Dark Matter, PoS ICRC2017 (2018) 929 [1711. 10971$].$

[17] R. L. Jaffe, Perhaps a Stable Dihyperon, Phys. Rev. Lett. 38 (1977) 195.

[18] BABAR collaboration, Search for a Stable Six-Quark State at BABAR, Phys. Rev. Lett. 122 (2019) 072002 [1810.04724].

[19] M. Raggi and V. Kozhuharov, Proposal to Search for a Dark Photon in Positron on Target Collisions at DAФNE Linac, Adv. High Energy Phys. 2014 (2014) 959802 [14 03.3041 ].

[20] P. Valente, Searching for Dark Photons with PADME, in La Thuile - Les Recoontres de physics de la valee D’Aoste, 2019.

[21] BELLE-II collaboration, The Belle II Physics Book, 1808.10567.

[22] G. Inguglia, BELLE II, in La Thuile - Les Recoontres de physics de la valee D'Aoste, 2019.

[23] S. N. Gninenko, D. V. Kirpichnikov, M. M. Kirsanov and N. V. Krasnikov, Combined search for light dark matter with electron and muon beams at NA64, Phys. Lett. B796 (2019) 117 [1903 07899 ]. 